\title{
Development of a prognostic pyroptosis-related gene signature for head and neck squamous cell carcinoma patient
}

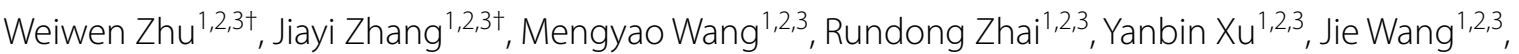 \\ Mengqi Wang ${ }^{1,2,3}$, Hang Zhang ${ }^{2,3}$ and Laikui Liu ${ }^{1,2,3^{*}}$ (])
}

\begin{abstract}
Objective: Head and neck squamous cell carcinoma (HNSCC) is a major threat to public health. Pyroptosis is a form of inflammatory programmed cell death that is still incompletely understood. The role of pyroptotic cell death in HNSCC remains to be fully defined. As such, the present study was developed to explore the potential prognostic utility of a pyroptosis-related gene (PRG) signature in HNSCC.
\end{abstract}

Methods: PRG expression patterns and the associated mutational landscape in HNSCC were analyzed, after which a 6-gene prognostic model was constructed through least absolute shrinkage and selection operator (LASSO) and Cox regression analyses using the TCGA dataset, followed by validation with two GEO datasets (GSE41643 and GSE65858). The relative expression of the genes in the prognostic model was assessed via RT-qPCR in tumor and paired adjacent normal tissue samples from a 32-patient cohort. Potential predictors of patient outcomes associated with this 6-gene model were identified through topological degree analyses of a protein-protein interaction network. Moreover, the prognostic value of NLRP3 as a predictor of HNSCC patient prognosis was established through immunohistochemical (IHC) analyses of samples from $176 \mathrm{HNSCC}$ patients. Lastly, in vitro studies were performed to further demonstrate the relevance of NLRP3 in the context of HNSCC development.

Results: Differentially expressed PRGs were able to readily differentiate between HNSCC tumors and normal tissues. Risk scores derived from the 6-gene PRG model were independent predictors of HNSCC patient prognosis, and genes that were differentially expressed between low- and high-risk groups were associated with tumor immunity. RT-qPCR assays also showed the potential protective role of NLRP3 in HNSCC patients. IHC analyses further supported the value of NLRP3 as a predictor of HNSCC patient outcomes. Invasion and migration assays demonstrated the potential role of NLRP3 in the inhibition of HNSCC development.

Conclusions: Overall, these results highlight a novel prognostic gene signature that offers value in the context of HNSCC patient evaluation, although additional research will be essential to elucidate the mechanisms linking these PRGs to HNSCC outcomes.

Keywords: Head and neck squamous cell carcinoma, Pyroptosis, Prognostic model, NLRP3

*Correspondence: my_yunkong@njmu.edu.cn

†'Weiwen Zhu and Jiayi Zhang contributed equally to this work ${ }^{1}$ Department of Basic Science of Stomatology, The Affiliated Stomatological Hospital of Nanjing Medical University, 136\# Hanzhong Road, Nanjing 210029, Jiangsu, China

Full list of author information is available at the end of the article

\section{Introduction}

Head and neck cancer is the sixth most prevalent form of cancer globally, with an estimated 830,000 diagnoses and 379,000 deaths occurring annually $[1,2]$. Over $90 \%$ of these cases are diagnosed as head and neck original author(s) and the source, provide a link to the Creative Commons licence, and indicate if changes were made. The images or other third party material in this article are included in the article's Creative Commons licence, unless indicated otherwise in a credit line to the material. If material is not included in the article's Creative Commons licence and your intended use is not permitted by statutory regulation or exceeds the permitted use, you will need to obtain permission directly from the copyright holder. To view a copy of this licence, visit http://creativecommons.org/licenses/by/4.0/. The Creative Commons Public Domain Dedication waiver (http://creativeco mmons.org/publicdomain/zero/1.0/) applies to the data made available in this article, unless otherwise stated in a credit line to the data. 
squamous cell carcinoma (HNSCC), which arises from the mucosal epithelium of the oral cavity, pharynx, and larynx $[3,4]$. Primary treatments for HNSCC patients include various combinations of surgery, radiotherapy, and chemotherapy depending on disease staging [5]. As there are no reliable approaches to systematically screening for this disease, a large percentage of HNSCC patients are diagnosed with advanced disease [6]. While there have been recent advances in treatment strategies for these patients, their 5-year survival rates remain low, underscoring the importance of developing new approaches to diagnosing and monitoring HNSCC in an individualized manner.

Biomarkers are specific indicators of particular physiological, pathological, or pharmacological processes that can enable effective patient identification and monitoring [7]. The advent of high-throughput sequencing technologies has spurred growing interest in the identification of specific biomarkers capable of guiding the prognostic evaluation and treatment of various cancers [8-10]. Many predictive biomarkers associated with HNSCC have been studied to date and may be of value as predictors of long-term patient prognosis and targeted treatment outcomes [11-13]. Most of these biomarkers, however, have yet to be subjected to any robust clinical validation or drug targeting efforts [14]. Prognostic biomarker models that are better able to predict HNSCC patient prognosis and treatment outcomes in a reliable manner are thus urgently needed to improve survival outcomes for affected individuals.

Pyroptosis is a form of inflammatory programmed cell death distinct from apoptosis [15]. Also known as cellular inflammatory necrosis, pyroptosis results in immunostimulatory molecule release from tumor cells [16]. At the molecular level, pyroptosis is associated with distinctive molecular features such as chromatin condensation, DNA fragmentation in the absence of DNA damage, and pore formation $[17,18]$. Pyroptotic cell death has been suggested to play contrasting roles as an inhibitor or promoter of oncogenic progression, and it is closely correlated with cellular migration and proliferation [1921]. Pyroptosis can be induced in response to specific microRNAs (miRNAs), chemotherapeutic drug treatments, or inflammasome activation, suppressing tumor growth [16]. Conversely, pyroptosis-related inflammatory cytokine production can give rise to a microenvironment that is better suited to tumor cell growth [22]. Pyroptosis-related gene (PRG) signatures can offer insight into prognostic outcomes across a range of cancer types [23, 24]. Treatment with TPL can activate GSDME-mediated pyroptosis by inhibiting the expression of HK-II in the mitochondria in HNSCC [25]. Although PRGs have been shown to have the prognostic value when predicting
HNSCC patient outcomes [26], PRG-mediated immune infiltration and the aberrant changes in the biological behavior of cancer cells remain poorly understood.

Herein, we compared PRG expression between control and HNSCC tumor tissue samples to establish the value of these genes as predictors of patient outcomes. A novel PRG-based prognostic model for HNSCC was then developed and validated through both bioinformatics analyses of extant datasets and additional clinical samples.

\section{Materials and methods \\ Data processing}

RNA-sequencing (RNA-seq) data and matching clinicopathological findings pertaining to 506 patients with HSCC were downloaded from The Cancer Genome Atlas (TCGA) on Aug 10, 2021 (Additional file 1: Table S1). Somatic and copy number variation (CNV) data were downloaded from the TCGA and UCSC Xena websites. RNA-seq and clinical data used for external validation were downloaded from the GEO database (ID: GSE41643 and GSE65858). R (v 4.0.1) and the R Bioconductor packages were used for all analyses.

\section{Differentially expressed PRG identification}

In total, the 33 PRGs listed in Additional file 2: Table S2 were identified based on prior reviews [16, 27]. Given that there were 31 annotated PRGs in the TCGA database, the differences in the expression of these genes between HNSCC patients and healthy controls were established using the 'limma' package. The Search Tool for the Retrieval of Interacting Genes (STRING) database was further utilized to construct a protein-protein interaction (PPI) network incorporating these PRGs based on a minimum interaction score of 0.7 .

\section{Functional enrichment analyses}

The R 'ggplot2' package was utilized to conduct Gene Ontology (GO) and Kyoto Encyclopedia of Genes and Genomes (KEGG) enrichment analyses.

\section{Prognostic PRG model development}

A Cox regression analysis was used to assess the prognostic value of PRGs in HNSCC, with 6 of these significantly prognostic genes being retained for a subsequent prognostic model development through a LASSO Cox regression analysis. HNSCC patients from the TCGA cohort were separated into low- and high-risk groups based upon the median risk score value in this cohort, with Kaplan-Meier analyses subsequently being used to compare overall survival (OS) outcomes between these groups. Validation of this model was performed using the GSE41643 and GSE65858 datasets, and risk scores were 
calculated using the same formula employed for TCGA patient analyses.

\section{Real-time quantitative PCR (qPCR) analysis}

RNA was extracted from cells using the Trizol reagent and reverse transcribed using the PrimeScript RT Reagent Kit (Takara Bio, Kusatsu, Japan). All qPCR analyses were performed in triplicate using the SYBR Green PCR Master Mix (Takara Bio) and detected using an Applied Biosystems 7900 Real-Time PCR System (Thermo Fisher Scientific, Waltham, MA, USA). The primer sequences used in this study are listed in Additional file 3: Supplementary Materials. Gene expression values were normalized to the endogenous control, and the $2^{-\Delta \Delta C t}$ method was used for the relative quantification of gene expression. Primers used were as follows: GAPDH: forward 5'-GAAGGTGAAGGTCGGAGTC- $3^{\prime}$ and reverse 5'-GAGATGGTGATGGGATTTC-3'; NLRP1: forward $5^{\prime}$-AGCTTCTGCTCGCCAATAAAG- $3^{\prime}$ and reverse 5'-CCAGGTATGGAGGGCTAGGT-3'; NLRP2: forward $5^{\prime}$-TTCTGCGTCAAGCACTGTCG- $3^{\prime}$ and reverse 5'-GGATCTCTCAACCTCGGCGT-3'; NOD2: forward $5^{\prime}$-CAATGACGATGCGGACACTG- ${ }^{\prime}$ and reverse 5'-GCTGAATGGGAAGACAAAGAGAA-3'; PLCG1: forward $5^{\prime}$-CTCTATGGAATGGAATTTCGCC- $3^{\prime}$ and reverse 5'-GGAGCCACCTCTCAATCTGC-3'; NLRP3: forward $5^{\prime}$-AGCACTAATCAGAATCTCACGCA- $3^{\prime}$ and reverse $5^{\prime}$-TGTCTAATTCCAACACCTGAAGC-3'; IL-6: forward $5^{\prime}$-TGCCAGCCTGCTGACGAA- $3^{\prime}$ and reverse 5'-AGCTGCGCAGAATGAGATGA-3'.

\section{Patients}

In total, 176 primary HNSCC patients undergoing surgical treatment at the Department of Oral and Maxillofacial Surgery of the Affiliated Stomatological Hospital of Nanjing Medical University between 2010 and 2015 were recruited for this study. WHO classification criteria were used for tumor grading, while UICC and American Joint Commission on Cancer (AJCC) criteria were used for clinical and TNM grading. The Nanjing Medical University Ethics Committee approved this study, which was consistent with the Declaration of Helsinki. All patients provided written informed consent to participate.

\section{Immunohistochemical (IHC) staining}

Paraffin-embedded HNSCC tumor tissue sections $(4 \mu \mathrm{m})$ were treated with xylene for deparaffinization, rehydrated with an ethanol gradient, and treated for 20 min with 3\% hydrogen peroxide. Following antigen retrieval, samples were blocked with normal goat serum and then probed overnight with anti- NLRP3 (Proteintech, 19771-1-AP). An HRP-polymer anti-rabbit Kit and a DAB Detection Kit (Fuzhou Maixin Biotech, Fuzhou, China) were then used to stain samples, and hematoxylin was employed for counterstaining. An ethanol gradient was then used to dehydrate samples, which were clarified with xylene and mounted using neutral gum.

\section{Pathological staining analyses}

Two pathologists independently analyzed pathological samples. NLRP3 staining was assessed based upon immunoreactivity score (IRS) values calculated as: IRS $=$ IS $\times$ PS. IS scoring was as follow: 0 , negative; 1 , weak; 2 , moderate; 3 , strong. PS scoring was as follows: 0 , negative; $1,<10 \% ; 2,11-50 \% ; 3,51-80 \% ; 4,>80 \%$ positive staining. Patients were then separated into low- and high-expression subgroups based on IRS scores of 0-4 and $>4$, respectively.

\section{Cell proliferation assay}

5-Ethynyl-2'-deoxyuridine (EdU) staining was used to assess proliferation. Briefly, cells were incubated with complete DMEM medium containing $50 \mu \mathrm{M}$ EdU (RiboBio) at $37{ }^{\circ} \mathrm{C}$ in a $5 \% \mathrm{CO}_{2}$ incubator for $2 \mathrm{~h}$. Then, cells were washed with $\mathrm{PBS}$, fixed with $4 \%$ paraformaldehyde for $30 \mathrm{~min}$, neutralized with $50 \mu \mathrm{L} 2 \mathrm{mg} / \mathrm{mL}$ glycine, and permeabilized using $0.5 \%$ Triton X-100. After washing with PBS, Apollo dye was added to each well, and then cells were incubated in the dark for $30 \mathrm{~min}$ at room temperature. Finally, Hoechst 33342 was applied for nuclear staining. Images were then captured using a fluorescence microscope (Leica Microsystems, Mannheim, Germany).

\section{Cell migration and invasion assays}

Cell migration and invasion assays in vitro were performed using wound healing and transwell assays. In the wound healing assay, cells were plated in six-well plates and grown to $90 \%$ confluence. Artificial wounds were created in the monolayer surface using a $10 \mu \mathrm{l}$ sterile pipette tip, and then the cells were then incubated in complete medium and allowed to migrate into the open wound area. Images of the same area of the wound were taken at 0,12 , and $24 \mathrm{~h}$ to determine the wound closure rate. Cell invasion assays were performed using Transwell inserts, and the Transwell inserts with the porous membrane (pore size $8 \mu \mathrm{m}$, Miliipore), which has been precoated with Matrigel (Corning, Bedford, MA, USA) for at least $1 \mathrm{~h}$ at $37^{\circ} \mathrm{C}$. Approximately $1 \times 10^{5} \mathrm{HN} 6$ cells or $2 \times 10^{5} \mathrm{Cal} 27$ cells were seeded per well in the upper chambers and incubated with complete medium in the lower chamber. After $24 \mathrm{~h}$, the Transwell chambers were fixed with 4\% PFA and stained with crystal violet (SigmaAldrich, St. Louis, MO, USA). Cells attached to the lower layer were then imaged (Olympus, Tokyo, Japan), every experiment was repeated for three times, and every chamber were counted in 5 randomly selected fields. 


\section{Results}

\section{Analysis of the PRG landscape in HNSCC}

We began by assessing CNVs evident in 31 annotated PRGs from the TCGA HNSCC dataset, revealing alterations to be present in all of these genes. Of these, the highest CNV amplification frequency was observed for GSDMC, while the highest frequency of deletion was observed for GPX4 (Fig. 1A). The chromosomal locations of these PRGs were next established (Fig. 1B). Of 506 patients in the TCGA HNSCC cohort, 124 (24.51\%) harbored somatic mutations in these PRGs. CASP8 was found to be mutated in $8 \%$ of these HNSCC patients, with missense and nonsense mutation being the most prevalent mutation type, thus suggesting a potential role of CASP8 in these HNSCC patients (Fig. 1C). Among all the mutations observed in identified PRGs, missense mutations were the most common (Fig. 1D), with $\mathrm{C}>\mathrm{T}$ mutations being the most frequently detected form of single nucleotide polymorphism (SNP) in these patients. Next, PRG expression levels were compared between HNSCC tumors and control tissues, leading to the identification of 21 differentially expressed genes (DEGs) (Fig. 2A), of which 18 (CASP1, CASP3, CASP5, CASP6, CASP8, GSDMB, GSDMD, GSDME, IL-1B, NLRC4, NLRP1, NLRP6, NLRP7, NOD1, PLCG1, PYCARD, SCAF11, and TNF) were upregulated in tumors and three (CASP9, ELANE, and IL-18) were downregulated (Fig. 2B). A PPI network was then constructed to explore interactions among these PRGs in HNSCC (Fig. 2C), and a PRG correlation network was generated with positive and negative correlations being shown in red and blue, respectively (Fig. 2D). Overall, in light of CASP8 exhibiting the highest mutation rate among these PRGs and its observed upregulation in tumor tissues, the detailed etiological role of CASP8 in HNSCC development may warrant further study.

\section{PRG-based HNSCC patient clustering and functional enrichment analyses}

To better understand the relationship between PRG expression and HNSCC, we conducted the consensus clustering of HNSCC patients from the TCGA dataset. Of tested values (2-10), we found that a clustering variable $(\mathrm{k})$ value of 2 was associated with maximal intragroup correlations while maintaining low intergroup correlations. As such, these 506 HNSCC patients were effectively separated into two PRG-based clusters (Fig. 3A). When gene expression and clinical profiles were compared between these two clusters ( $C 1$ and $\mathrm{C} 2$ ), gender was the only discrepant feature (Fig. 3B). Overall survival (OS) did not differ significantly when comparing patients in these clusters $(\mathrm{P}=0.554$, Fig. 3C).

GO enrichment analyses of these 31 PRGs primarily revealed them to be associated with the positive regulation of cytokine production and interleukin-1 production (Fig. 3D). KEGG enrichment analyses further highlighted a role for these PRGs in the NOD-like receptor signaling and Salmonella infection pathways (Fig. 3E).

\section{PRG-associated prognostic model development}

Data pertaining to 500 HNSCC patients for whom survival outcomes were available in the TCGA cohort were used to guide prognostic model development. Initial univariate Cox regression analyses identified 6 PRGs (IL-6, NLRP1, NLRP2, NLRP3, NOD2, and PLCG1) that were significantly associated with patient survival $(\mathrm{P} \leq 0.1)$, of which IL-6 and NLRP2 were associated with risk increases $(\mathrm{HR}>1)$, whereas NLRP1, NLRP3, NOD2, and PLCG1 were protective $(\mathrm{HR}<1)$ (Fig. 4A). Next, least absolute shrinkage and selection operator (LASSO) (Fig. 4B, C) and Cox regression analyses (Fig. 4D) were utilized to construct a 6-gene model based on an optimal $\lambda$ value. This model was as follows: risk score $=(0.0871 *$ IL6 exp. $)+(0.3764 *$ NLRP1 exp. $)+(0.0595 * \mathrm{NLRP} 2 \quad$ exp. $)+(0.3820 * \mathrm{NLRP} 3$ exp. $)+\left(0.2357^{*}\right.$ NOD2 exp. $)+(0.1371 *$ PLG1 exp. $)$. These HNSCC patients were then stratified into lowand high-risk groups based upon median risk score values (Fig. 4E). In a principal component analysis (PCA), low- and high-risk patients were readily separated into two clusters (Fig. 4F). High-risk HNSCC patients exhibited poorer OS outcomes relative to low-risk patients $(\mathrm{P}<0.001)$ (Fig. 4G). Time-dependent receiver operating characteristic (ROC) analyses were also utilized to assess the specificity and sensitivity of this model, revealing respective area under the ROC curve (AUC) values for 1-, 2-, and 3-year survival outcomes of $0.610,0.654$, and 0.703 (Fig. 4H). In summary, based on the Cox regression analysis, we further demonstrated the potential protective roles of NLRP1, NLRP3, and NOD2 as predictors of patient outcomes in a prognostic model.

\section{External risk signature validation}

To validate the risk signature developed above, data pertaining to 367 HNSCC patients from the GSE41643 and GSE65858 datasets were utilized. Batch effects between these datasets were reduced with the 'ComBat' algorithm. These patients were then separated into high-risk $(n=190)$ and low-risk $(n=177)$ groups based upon the median risk scores from the TCGA cohort (Fig. 5A). PCA analysis revealed that these 


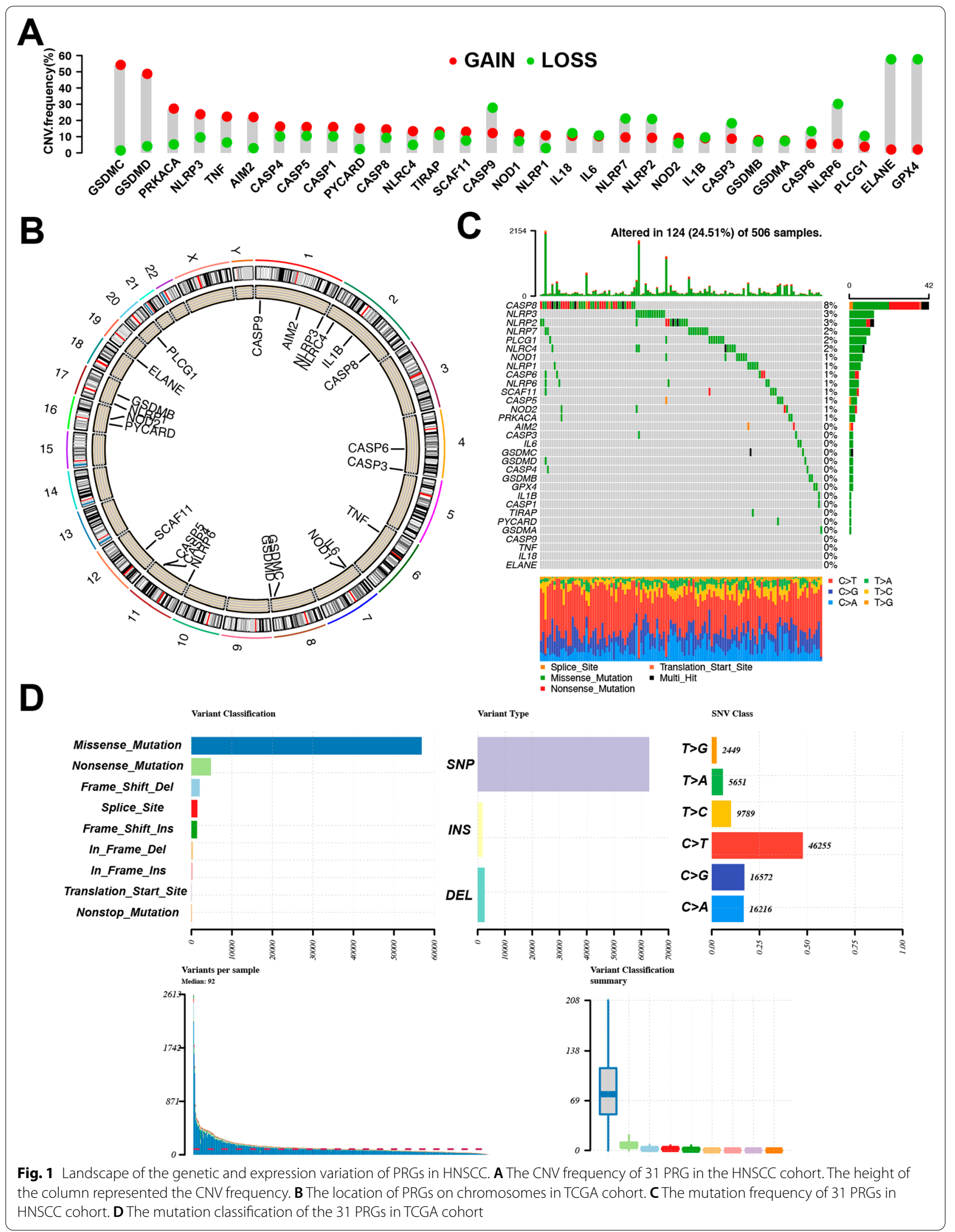



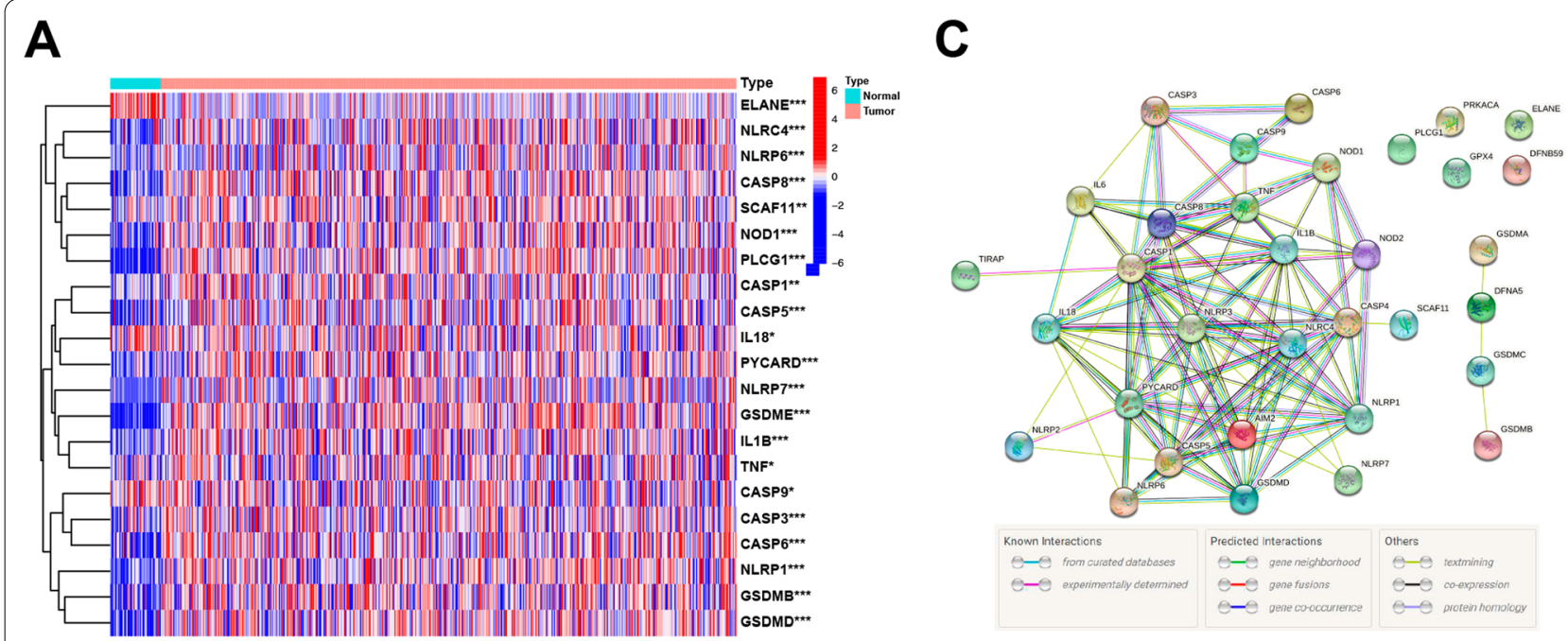

B
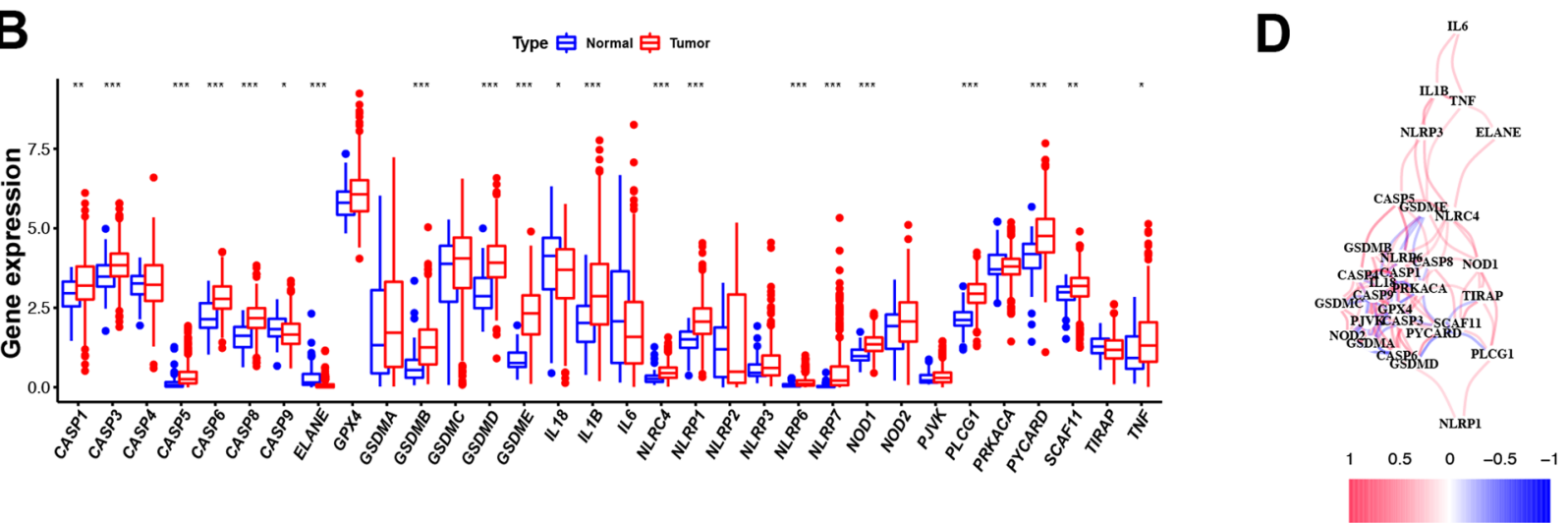

Fig. 2 Expressions of the 31 pyroptosis-related genes and the interactions among them. A Heatmap (blue: low expression level; red: high expression level) of the pyroptosis-related genes between the normal ( $\mathrm{N}$, brilliant blue) and the tumor tissues (T, red). B The expression of 31 PRG in HNSCC and normal tissues, Tumor, red; Normal, blue. The upper and lower ends of the boxes represented the interquartile range of values. The lines in the boxes represented median value. C PPI network showing the interactions of the pyroptosis-related genes (interaction score $=0.7$ ). $\mathbf{D}$ The correlation network of the pyroptosis-related genes (red line: positive correlation; blue line: negative correlation. The depth of the colors reflects the strength of the relevance). $P$ values were showed as: ${ }^{*} P<0.05 ;{ }^{* *} P<0.01 ;{ }^{* *} P<0.001$

two subgroups were appropriately separated from one another (Fig. 5B). Significant differences in OS were evident between low- and high-risk HNSCC patient groups $(P=0.002)$ (Fig. $5 \mathrm{C})$. ROC curve analyses further confirmed the predictive accuracy of this model, with respective AUC values pertaining to 1-, 2-, and 3 -year survival outcomes of $0.611,0.601$, and 0.558 (Fig. 5D).

\section{Analysis of risk score prognostic value and predictive nomogram construction}

Univariate and multivariate analyses were next performed to explore the prognostic utility of the risk score developed above. In univariate analyses, this risk score was able to independently predict survival outcomes in the TCGA and GEO datasets (TCGA, $\mathrm{HR}=2.060$; GEO, $\mathrm{HR}=1.714$ ) (Fig. 6A, D). In multivariate analyses, risk scores were similarly able to independently predict HSCC patient prognosis (TCGA, HR =1.977, GEO, HR: 1.910) (Fig. 6B, E). When a heatmap was constructed incorporating clinical findings from the TCGA (Fig. 6C) and GEO (Fig. 6F) cohorts, we observed diverse gender distributions between low- and high-risk patients in GEO cohorts $(\mathrm{P}<0.05)$. We then constructed a nomogram incorporating all independent prognostic variables from multivariate regression analyses, and we found that this model was able to effectively predict 1-, 


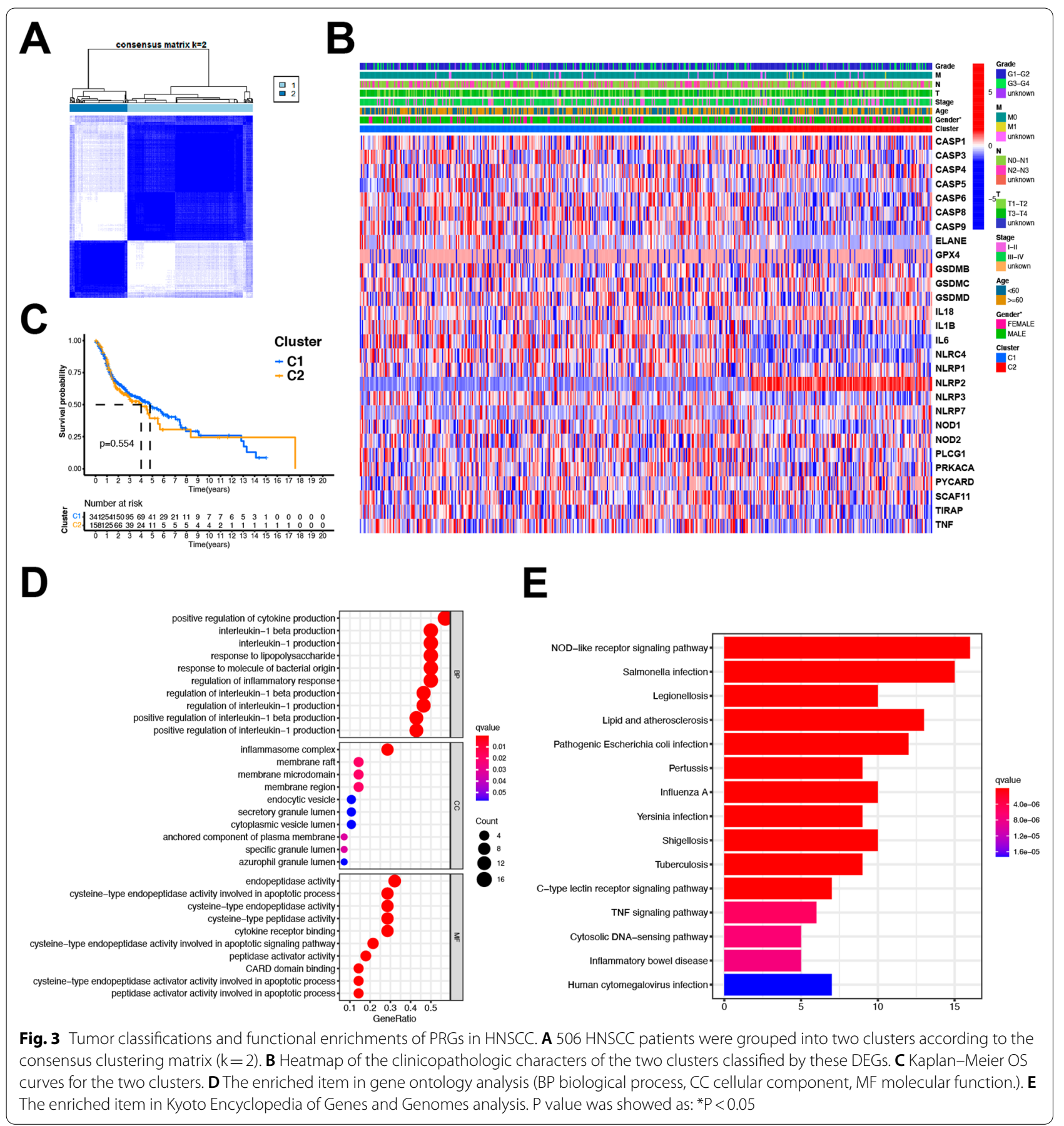

3-, and 5-tear OS in both GEO and TCGA cohorts (Fig. 6G-I). All these results further validated the accuracy of our model in the context of HNSCC patient prognostic evaluation.

\section{The relationship between prognostic PRGs and immune} functionality

Pyroptosis has been reported to be associated with the infiltration of the immune cells in various diseases. For example, PRG-induced macrophage infiltration was shown to be related to the release of inflammatory mediators, thereby inducing acute liver failure. Moreover, in the pyroptosis-activated immune 

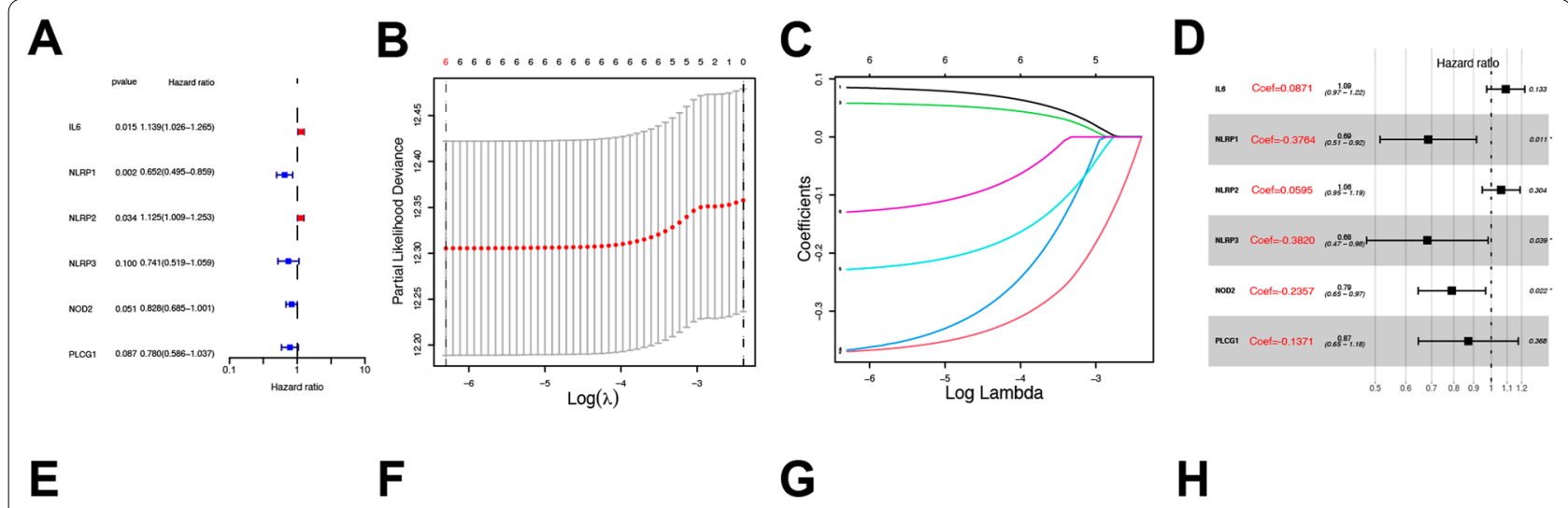

$\mathbf{F}$

G

H
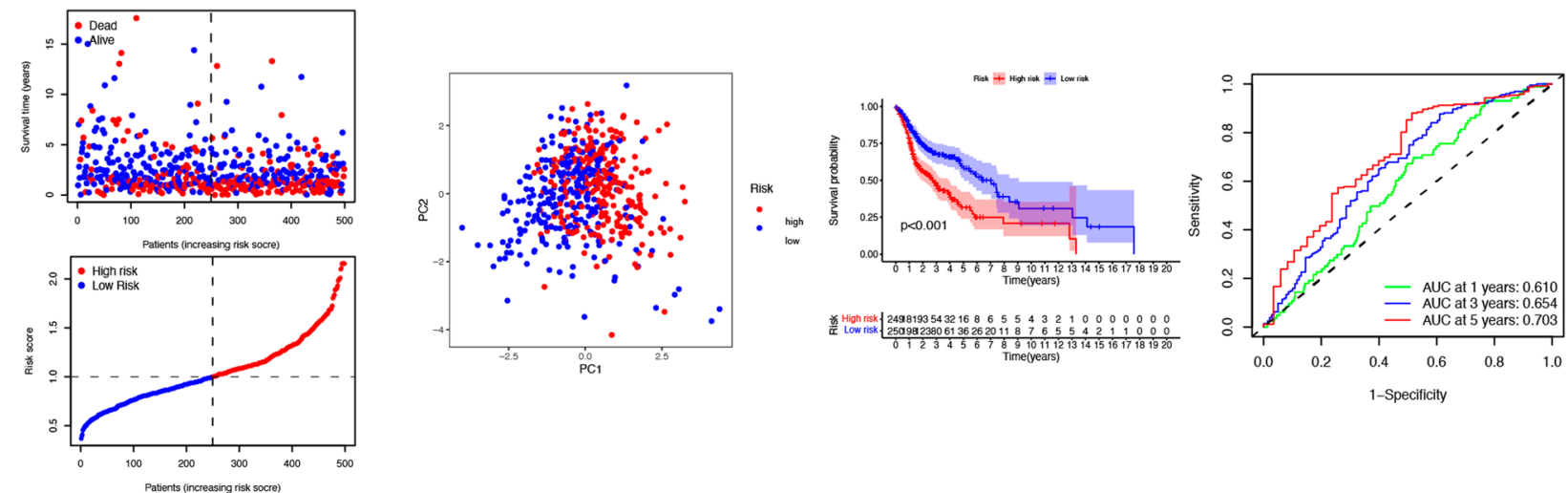

Fig. 4 Construction of the risk signature in TCGA cohort. A Univariate cox regression analysis of OS for each pyroptosis-related gene, and 6 genes with $\mathbf{P} \leq 0.1$. $\mathbf{B} L A S S O$ regression of the 6 OS-related genes. C Cross-validation for tuning the parameter selection in the LASSO regression. D Cox regression analysis for constructing the 6-gene prognostic model. E The survival status for each patient (low-risk population: on the left side of the dotted line; high-risk population: on the right side of the dotted line) (upper panel). Distribution of patients based on the risk score (lower panel). F PCA plot for HNSCC based on the risk score. G Kaplan-Meier curves for the OS of patients in the high- and low-risk groups. H ROC curves demonstrated the predictive efficiency of the risk score

microenvironment, CD8 $+\mathrm{T}$ cells and NK cells can both contribute to the generation of anti-tumor immunity $[28,29]$. To better understand the crucial role of PRGs in the development of the tumor immune microenvironment, single-sample gene set enrichment analysis (ssGSEA) scores were next used to compare enrichment scores pertaining to 16 immune cell types and 13 immune-related pathways between high- and low-risk HNSCC patients in the TCGA and GEO validation cohorts used above. High-risk patients were found to exhibit decreased iDC infiltration in the TCGA cohort (Fig. 7A), while NK cell infiltration was increased and macrophage infiltration was decreased in high-risk patients from the GSE65858 cohort (Fig. 7B). No significant differences in immune status were evident in the TCGA or GSE41643 cohorts (Fig. 7D, F), while cytolytic activity and inflammation-promoting functionality were enriched among high-risk individuals in the GSE65858 cohort (Fig. 7E).

When correlations between the 6 prognostic PRGs defined above and immune infiltration were assessed with the TIMER database, NLRP3 was found positively correlated with B cell, CD8 $+\mathrm{T}$ cell, CD4 $+\mathrm{T}$ cell, macrophage, neutrophil, and dendritic cell abundance (Fig. 8).

\section{Assessment of differential PRG expression in HNSCC patients}

Next, we recruited an independent cohort of 32 HNSCC patients consisting of 13 females and 19 males (40-66 years old), and sampled tumor tissues as well as paired normal epithelial tissues from these individuals for RT-qPCR analysis. After evaluating gene expression 


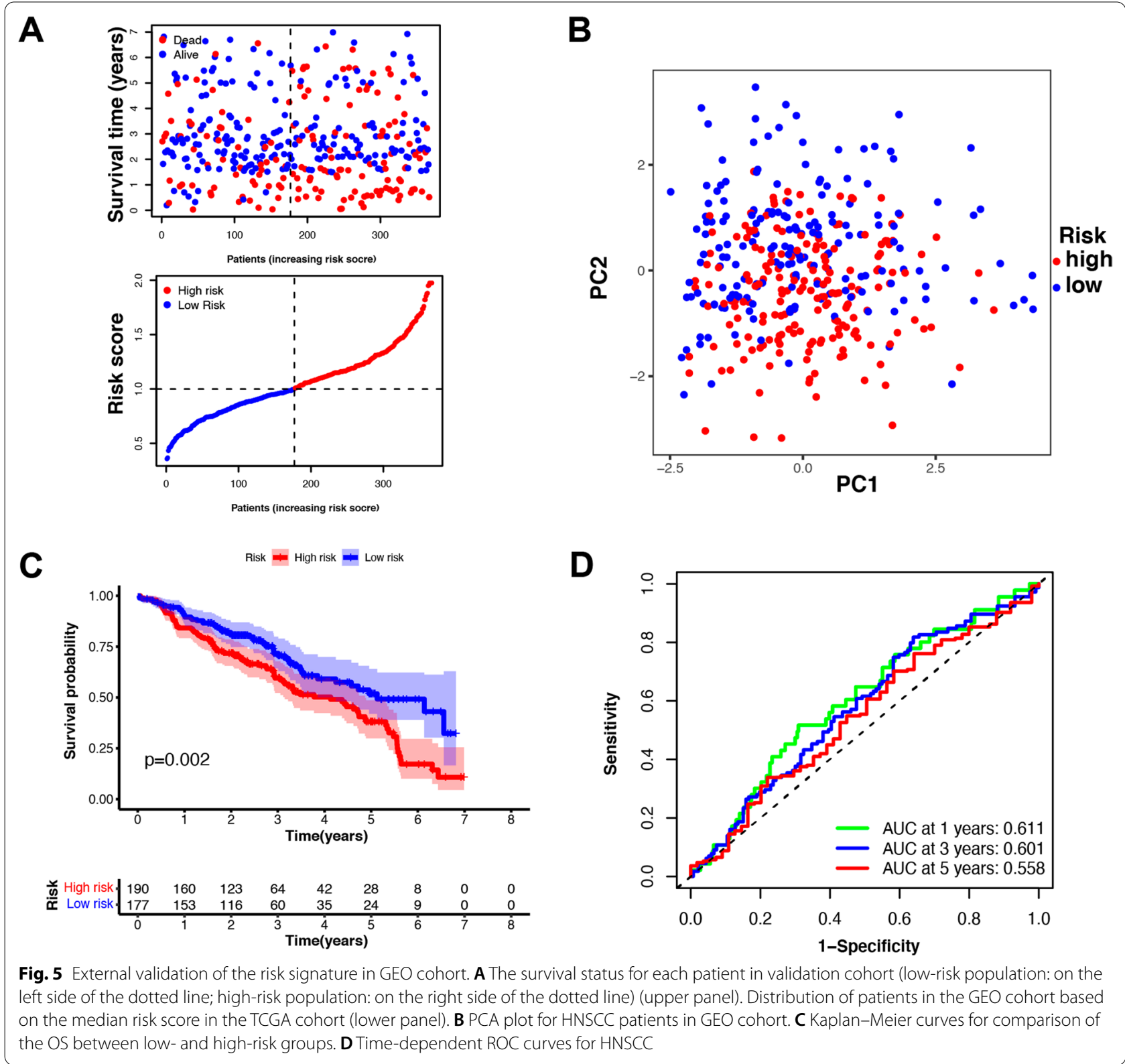

in our prognostic model, we demonstrated that NLRP2 and NLRP3 were significantly downregulated in HNSCC tissues in this patient cohort (Fig. 9). Given that NLRP2 was significantly associated with increased risk level $(H R>1)$ and NLRP3 was a protective gene $(\mathrm{HR}<1)$ (Fig. 4A), our results further supported the independent prognostic value of NLRP3 when predicting HNSCC patient outcomes.

\section{Lower expression of NLRP3 is related to a poorer HNSCC patient prognosis}

Topological degree analyses of the 31 PRGs in the PPI network developed above led to the selection of the top 8 of these genes, of which only NLRP3 was included in our prognostic gene signature (Fig. 10A). Given that the hazard ratio of NLRP3 in the prognostic model was 0.68 $(\mathrm{P}=0.039)$, we additionally conducted IHC analyses of NLRP3 staining in samples from 176 patients with HNSCC (Fig. 10B), the results demonstrated that the NLRP3 was significantly correlated with tumor size $(\mathrm{P}=0.023)$. However, there was no significant relationship between NLRP3 


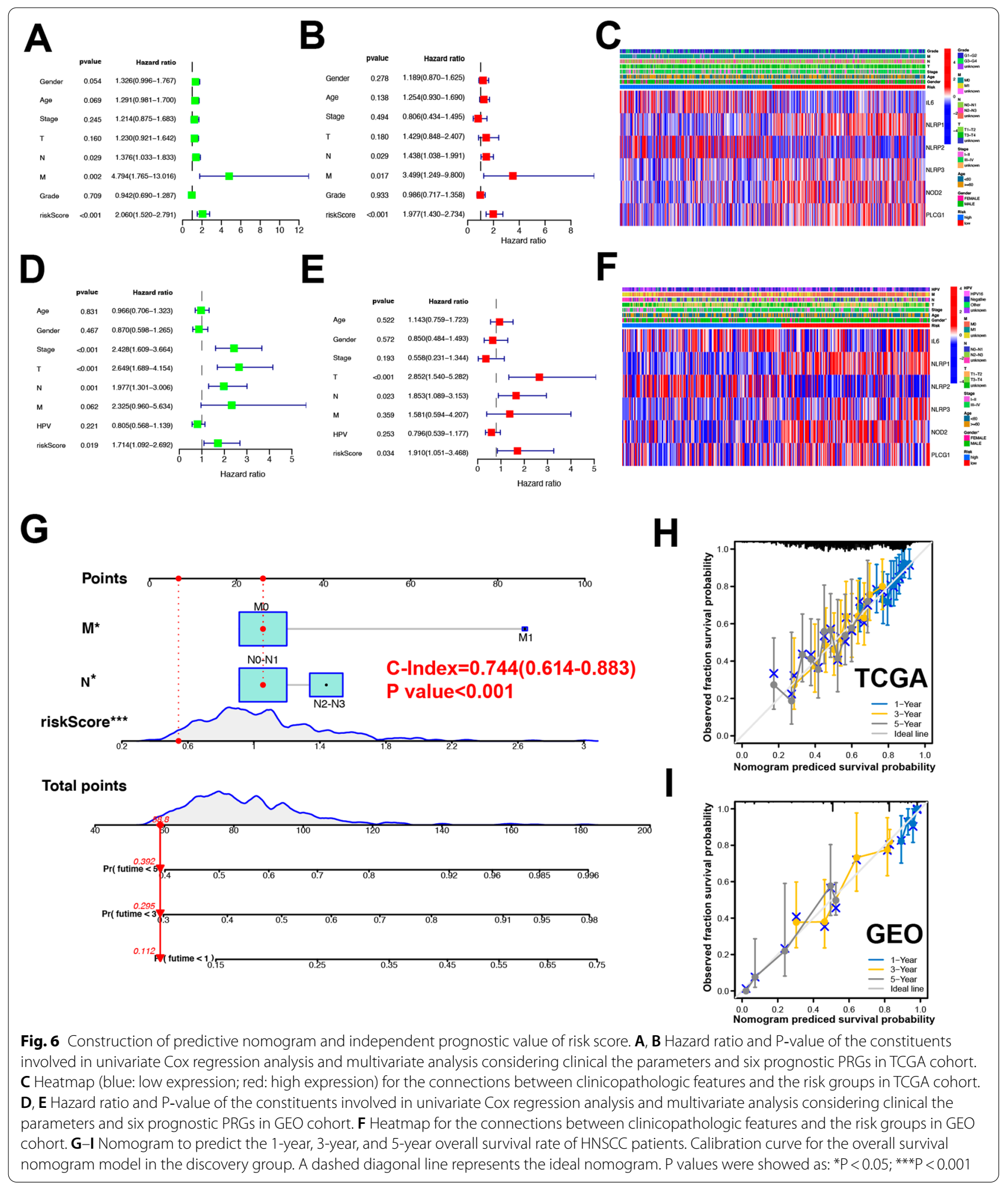

expression and gender, age, tumor location, distant metastasis, pathological grade, infiltration, clinical stage, or recurrence (Additional file 4: Table S3). In addition, the lower expression level of NLRP3 was associated with the poor survival of HNSCC patients (Fig. 10C, D). Together, 


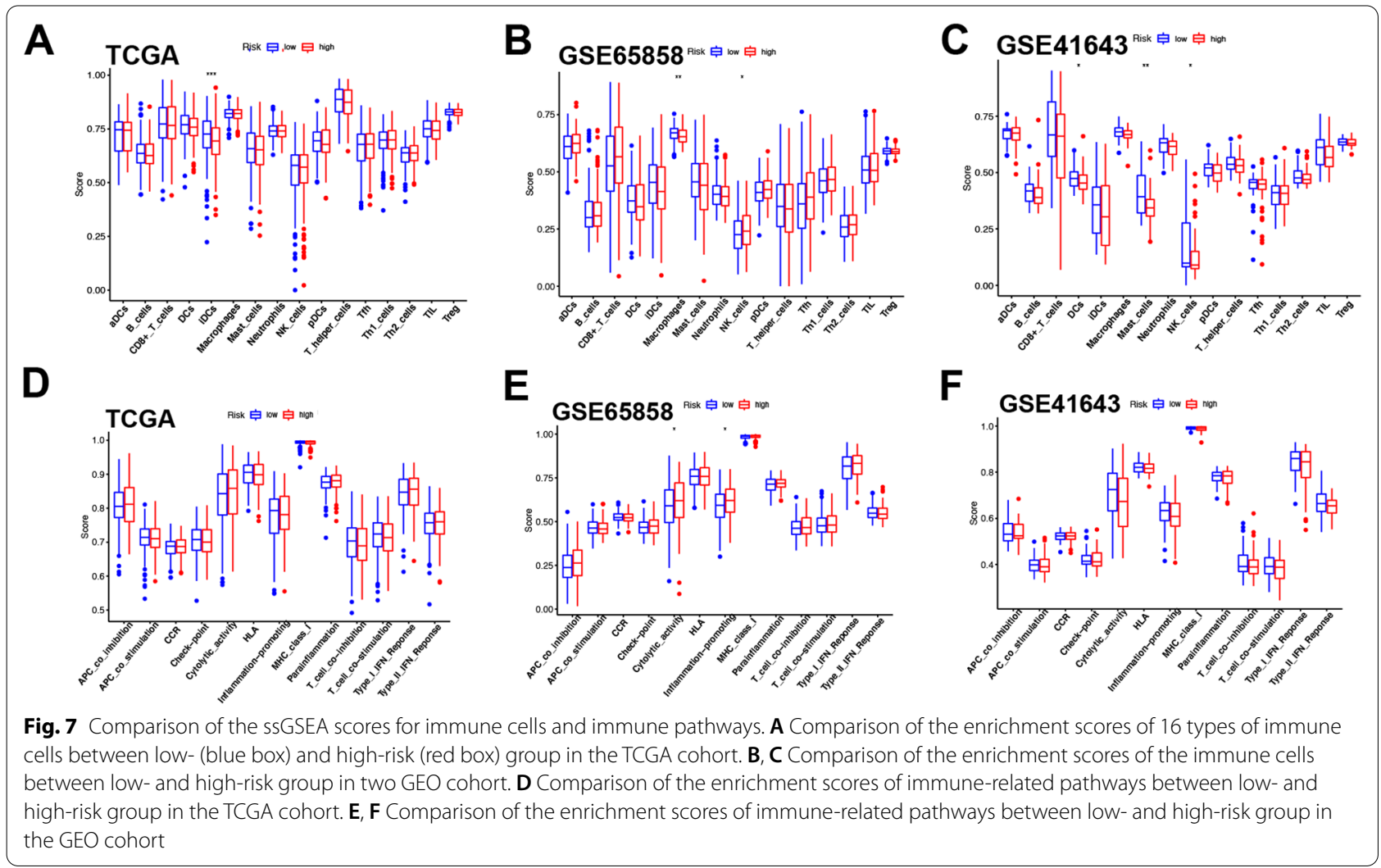

these findings confirm the prognostic utility of NLRP3 as a predictor of HNSCC patient survival.

\section{NLRP3 inhibits the invasion and migration of HNSCC cell lines}

Next, to demonstrate the effects of NLRP3 in the context of HNSCC development, we performed cell proliferation, invasion, and migration assays after knocking down or overexpressing NLRP3 in HN6 and Cal27 cell lines. These results indicated that the modulation of NLRP3 expression levels did not significantly affect cellular proliferation (Fig. 11A-C). However, Transwell assays demonstrated that NLRP3 knockdown resulted in enhanced cell invasion (Fig. 11D, F), whereas the overexpression of NLRP3 inhibited the invasion of HN6 and Cal27 cells (Fig. 11E, G). Wound healing assays also revealed that NLRP3 significantly inhibits cellular migration (Fig. $11 \mathrm{H})$. Collectively, these results suggested that NLRP3 can act as a tumor suppressor via inhibiting the invasion and migration of HNSCC cells.

\section{Discussion}

Normal physiological homeostasis is dependent on maintaining a balance between cellular proliferation, death, and differentiation [16]. While apoptosis and necrosis were once believed to be the primary forms of cell death, pyroptosis, autophagy, and other mechanisms have also been shown to mediate a loss of cellular viability [30]. Pyroptotic cell death is highly inflammatory and plays a role in a variety of pathogenic processes [31, 32]. Inflammasome activation initiates pyroptosis by recruiting caspase-1. Gasdermin D (GSDMD) serves as a caspase-1/11 substrate, inducing pyroptotic cell death owing to its ability to promote non-selective pore formation within the plasma membrane, subsequently driving cellular swelling, rupture, and the release of proinflammatory factors such as HMGB1, ATP, and IL-1 $\beta[16,18,33]$. The relationship between pyroptosis and cancer is complex, given that this form of cell death can both drive tumor progression and impair antitumor immunity [18], while also inhibiting oncogenesis [34]. The mechanisms whereby PRGs affect HNSCC progression and survival outcomes remain to be clarified. Herein, we evaluated expression levels of 31 different PRGs in samples from HNSCC patients in the TCGA database, revealing 21 of these genes to be differentially expressed in HNSCC including 18 that were upregulated in this oncogenic setting. GO and KEGG analyses demonstrated a link between PRGs and cytokine production, IL-1 production, NLRP signaling, and Salmonella infection. These functional pathways were closely associated with HNSCC oncogenesis and progression. Salmonella infection, for example, can 


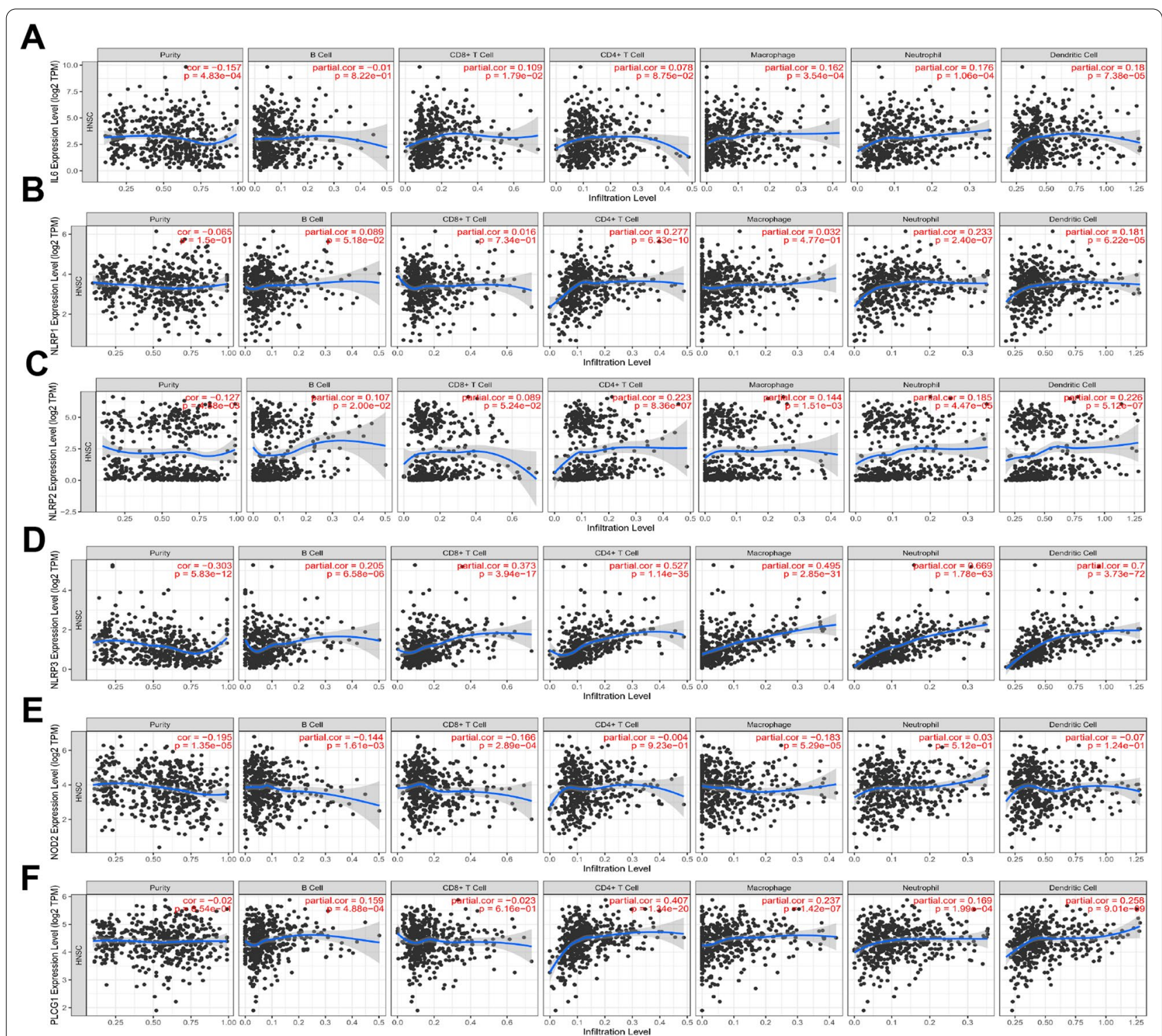

Fig. 8 The association between six prognostic PRGs and immune infiltration (TIMER). The association between the abundance of immune cells and the expression of (A) IL-6, (B) NLRP1, (C) NLRP2, (D) NLRP3, (E) NOD2 and (F) PLCG1 in HNSCC

promote TLR4/ MyD88 pathway activation and consequent increases in macrophage and neutrophil infiltration [35]. In OSCC, targeting of the ROS/NLRP3 inflammasome/IL-1 $\beta$ signaling pathway has been suggested to improve outcomes associated with 5-FU adjuvant chemotherapy [36]. These data suggest that in-depth PRG studies may offer improved insight into antitumor immunity and inflammation.

HNSCC patients exhibit poor long-term survival outcomes, underscoring the need for more reliable biomarkers of long-term patient prognosis and treatment outcomes. TNM stage, vascular invasion, and other traditional clinicopathological biomarkers have yielded unsatisfactory outcomes when used to gauge patient prognosis [37]. RNA-seq and other high-throughput sequencing technologies have led to the identification of a wide variety of prognostic biomarkers associated with different cancers [38, 39]. Recent work has shown individual biomarkers are ill-suited to gauging cancer patient prognosis. A 4-gene immune-related biomarker signature (PVR, TNFRSF12A, IL21R, and SOCS1) may, together with other clinicopathological metrics, offer value as a means of assessing HNSCC patient prognosis [40]. Wang et al. assessed patterns of gene expression from 771 HNSCC patients in the TCGA and GEO databases, leading to the development of a 6-gene 

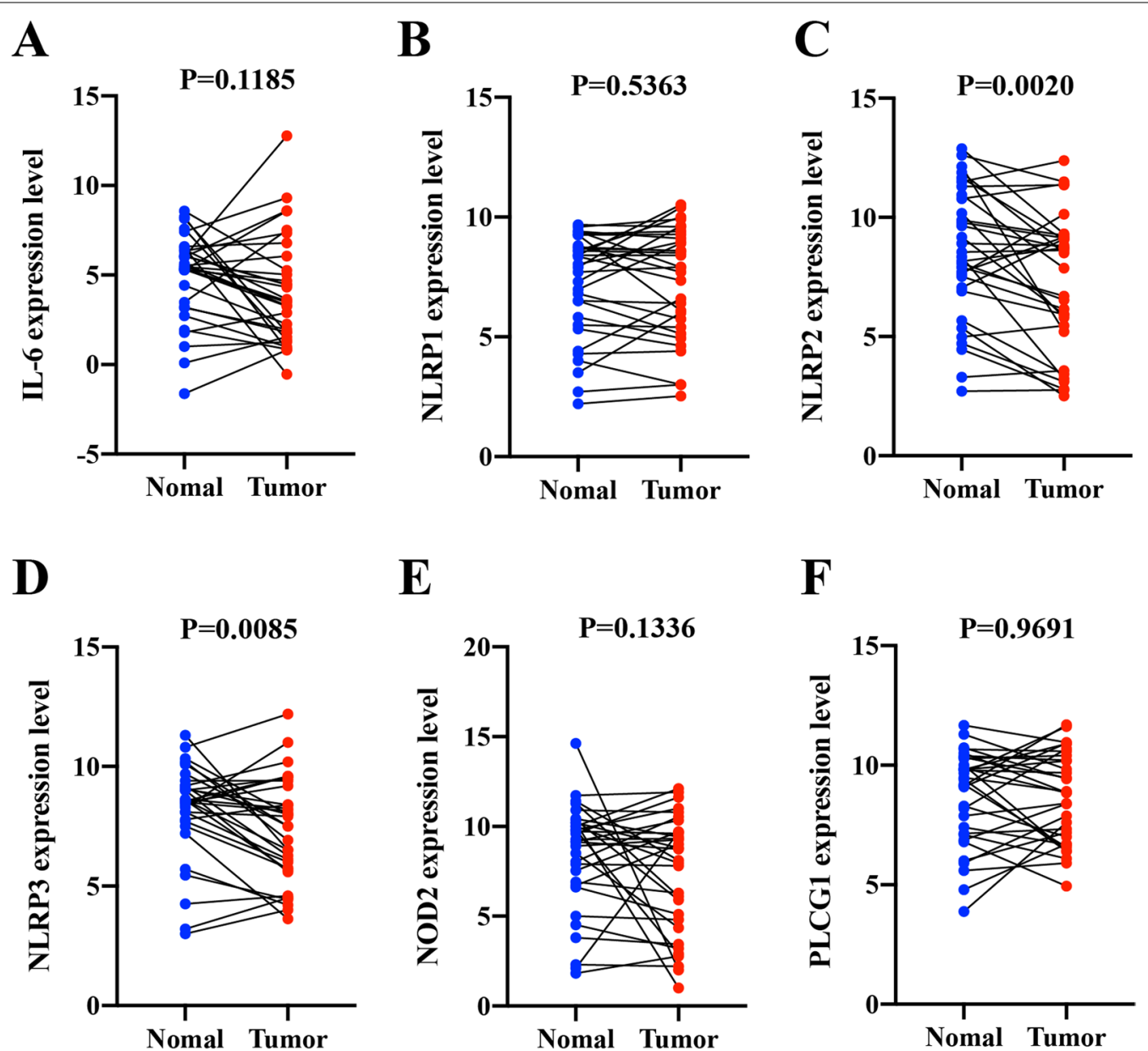

Fig. 9 RT-qPCR analysis of six genes in prognostic model. Relative mRNA level of (A) IL-6, (B) NLRP1 (C) NLRP2 (D) NLRP3 (E) NOD2 and (F) PLCG1 were assessed by RT-qPCR (Data was presented as mean \pm SD)

prognostic risk signature that was able to independently predict patient survival [41]. In a similar vein, we herein assessed the prognostic value of PRGs in HNSCC. We ultimately employed a LASSO Cox regression approach to construct a 6-PRG (IL-6, NLRP1, NLRP2, NLRP3, NOD2, and PLCG1) prognostic risk signature. Risk scores derived from this model were able to effectively stratify patients into low- and high-risk cohorts. Highrisk patients exhibited worse survival outcomes than did low-risk patients. These findings thus confirm the prognostic value of this novel PRG risk signature, highlighting a novel approach to predicting HNSCC patient outcomes.

Cell death plays a central role in diverse pathological processes [42], with pyroptosis functioning as an inflammatory type of caspase-mediated cell death that can modulate the immunogenic potential of specific cancers [43]. Such immunogenicity is of critical importance in the context of tumor immunotherapy owing to the ability of tumor cells to activate a variety of immunosuppressive pathways within the local tumor microenvironment [44]. Pyroptosis can impact immune cell composition and associated immunological pathway activation to alter the processes governing tumorigenesis. Herein, we compared the immunological status of patients in our low- and high-risk groups, and assessed the relationship between the levels of difference prognostic PRGs in HNSCC (IL-6, NLRP1, NLRP2, NLRP3, NOD2, and PLCG1) and immune cell infiltration. The results of these analyses suggest that this prognostic gene signature may have important implications for immunotherapy treatment planning. NK cell infiltration was related to the activation of pyroptosis [45] and alternatively differentiated macrophage (M2 macrophage) infiltration was associated with the decrease of inflammation and pyroptosis [46]. Interestingly, In the present study, we noted that NK cell infiltration was increased and macrophage infiltration was decreased in high-risk patients from the 


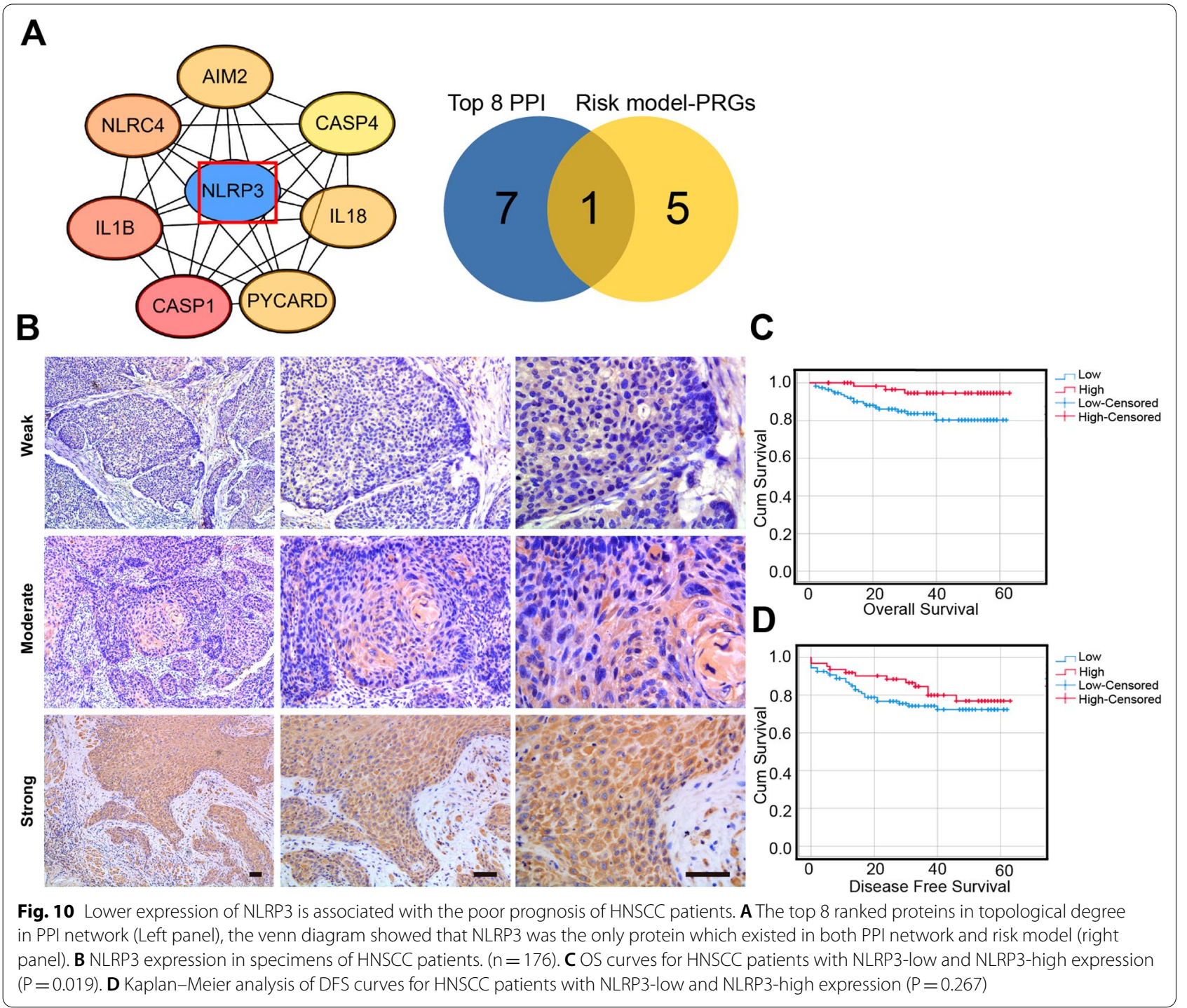

GSE65858 cohort, these results are also in line with the previous studies $[28,29]$, indicating the distinct role of macrophages and NK cells in cancer development, and suggesting the potential role of immune cell-mediated activation of pyroptosis in affecting HNSCC patients outcome.

Among the 6 genes in our prognostic model, NLRP3 was positively correlated with immune infiltration in the TIMER database. The activation of NLRP3 has previously been reported in the context of metabolic changes, mitochondrial dysfunction, and the disassembly of the Golgi compartment. The NLRP3 inflammasome can also promote the caspase-1-dependent release of IL-1 $\beta$ and IL-18, which are inflammatory cytokines, in addition to activating downstream pyroptotic signaling mechanisms [47]. As the best-characterized inflammasome, NLRP3 is known to be associated with the infiltration of various types of the immune cells and the induction of immune cell-mediated tumor suppression in gastric cancer, hepatic cancer, and lymphoma [48-50]. Interestingly, our

(See figure on next page.)

Fig. 11 NLRP3 inhibits the invasion and migration of HNSCC cells. Small interferon RNAs (Si-RNAs) and pcDNA 3.1 plasmids were used to knockdown or overexpress (OE) the NLRP3 expression. A-C EdU assay was used to assess the proliferation of HNSCC cell lines. scale bar $=50 \mu \mathrm{m}$. D-G Transwell assays of HN6 and Cal27 cell lines were used to determine the invasion of HNSCC cells. H Wound healing assays of HN6 and Cal27 cell lines $\left(\mathrm{OE}\right.$, overexpression; Data was presented as mean $\left.\pm \mathrm{SD},{ }^{*} \mathrm{P}<0.05,{ }^{* *} \mathrm{P}<0.01\right)$ scale bar $=100 \mu \mathrm{m}$ 


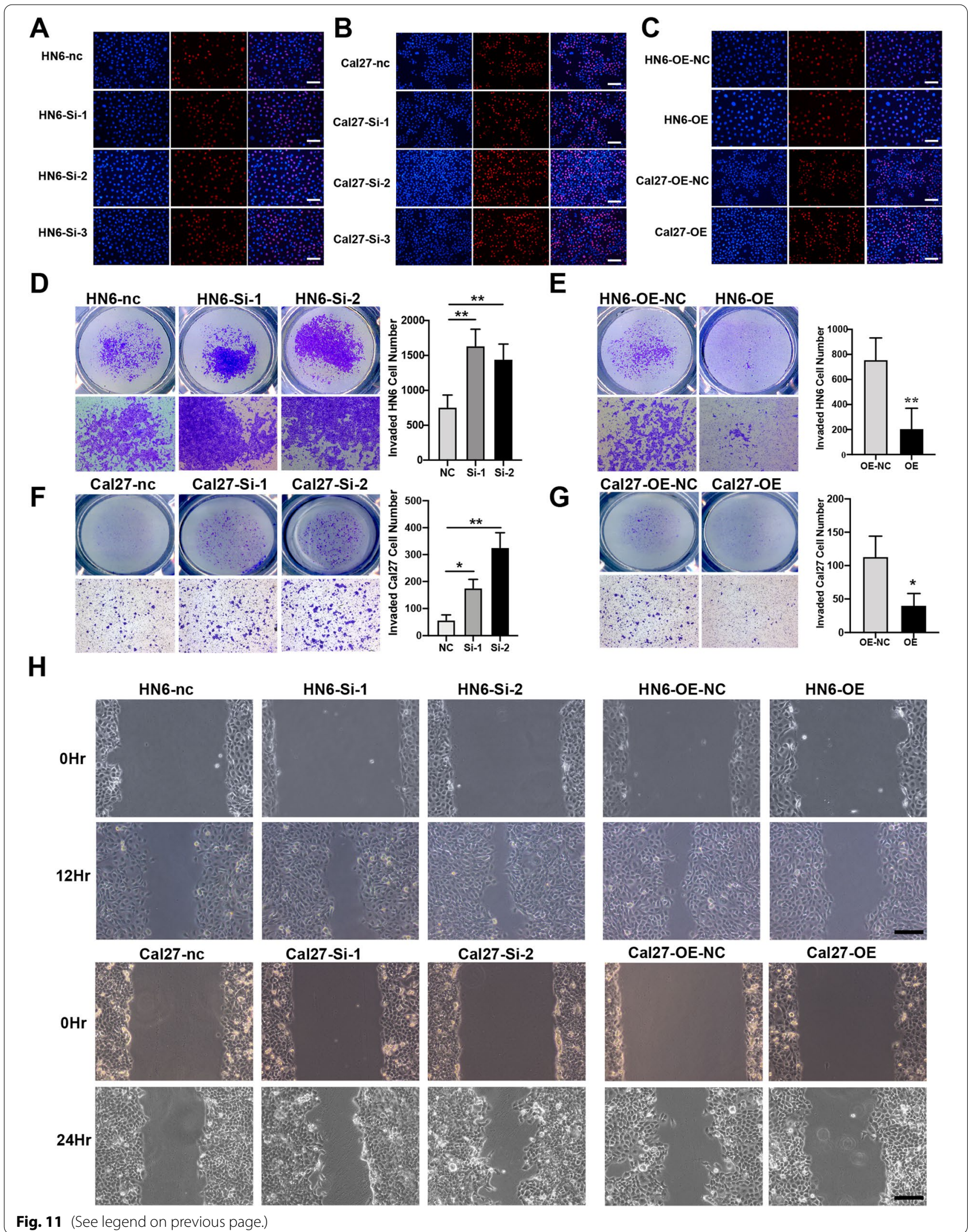


results are in line with those of previous studies, confirming the dual role of pyroptosis. Based on the Cox multivariate regression analysis of NLRP3 in our risk model, we posited that NLRP3 might act as a potential predictor of HNSCC patient outcomes $(\mathrm{HR}=0.68, \mathrm{P}=0.039)$. In line with these reports, the results of our PPI network and survival analyses suggested that NLRP3 may be a valuable predictor of prognosis among HNSCC patients. Relative to another similar study [26], we developed a distinct PRG-based prognostic model and highlighted the potential role of NLRP3 as a predictor of HNSCC patient outcomes, while finding that lower levels of NLRP3 expression detected via IHC staining were associated with a worse prognosis. In vitro studies of invasion and migration activity also underscored the protective role of NLRP3 in the inhibition of HNSCC development. Overall, these data demonstrate the value of NLRP3 as a PRG that is independently associated with HNSCC patient survival and cancer development.

\section{Conclusions}

In conclusion, the results of this study suggest that many differentially expressed PRGs were closely linked to the pathogenesis of HNSCC. Risk scores derived from our 6-gene PRG model were independent predictors of HNSCC patient outcomes, and genes that were differentially expressed between low- and high-risk groups were associated with tumor immunity. RT-qPCR analyses of tumor tissues also supported the protective role of NLRP3 in the context of HNSCC development, while IHC analyses further highlighted the value of NLRP3 as a predictor of HNSCC patient prognosis, and the expression of NLRP3 was additionally shown to affect the invasion and migration of HNSCC cells. Overall, these data highlight a novel gene signature that can be leveraged to predict survival outcomes in individuals with HNSCC, offering a foundation for future analyses of the link between PRGs and this cancer type.

\footnotetext{
Abbreviations

HNSCC: Head and neck squamous cell carcinoma; PRGs: Pyroptosis-related genes; RNA-seq: RNA-sequencing; TCGA: The Cancer Genome Atlas; GEO: Gene expression omnibus; PPI: Protein-protein interaction; STRING: Search Tool for the Retrieval of Interacting Genes; GO: Gene ontology; BP: Biological process; CC: Cellular component; MF: Molecular function; KEGG: Kyoto Encyclopedia of Genes and Genomes; IHC: Immunohistochemistry; ssGSEA: Single-sample gene set enrichment analysis; RT-qPCR: Real-time-quantitative PCR; OE: Overexpression; EdU: 5-Ethynyl-20-deoxyuridine.
}

\section{Supplementary Information}

The online version contains supplementary material available at https://doi.org/ 10.1186/s12935-022-02476-3.

Additional file 1: Table $S 1$.

Additional file 2: Table S2.
Additional file 3: Supplementary Materials.

Additional file 4: Table S3.

\section{Acknowledgements}

Thanks to Dr. Fang Shao for assisting us with statistical data presentation. We also thank Dr. Boxin Zhang for his kindly assistance of bioinformatic analysis.

\section{Authors' contributions}

WWZ and JYZ have the same contribution in this work. WWZ and JYZ analyzed the data and wrote paper. LKL applied the funding and designed the experiments. WWZ, MYW and HZ performed the experiments. WWZ, RDZ, MQW, JW and LKL edited the paper, YBX revised the manuscript. All authors contributed to the article and approved the summited version. All authors have read and approved the final manuscript.

\section{Funding}

This work was supported by a Grant from the National Natural Science Foundation of China (NSFC, No. 81972536), Key Project of Health Commission of Jiangsu Province (No. K2019013), the Natural Science Foundation of Jiangsu Higher Education Institutions of China (No. 20KJA320003), A Project Funded by the Priority Academic Program Development of Jiangsu Higher Education Institutions (PAPD, 2018-87), and Postgraduate Research \& Practice Innovation Program of Jiangsu Province (KYCX20_1409).

\section{Availability of data and materials}

The datasets used during the current study are available from the corresponding author on reasonable request.

\section{Declarations}

\section{Ethics approval and consent to participate}

The study was approved by the Ethics Committee of the Nanjing Medical University and samples were obtained with written informed consent from all patients, and all informed consent were conducted in accordance with the Declaration of Helsinki.

\section{Consent for publication}

Not applicable.

\section{Competing interests}

The authors declare that they have no conflict of interest.

\section{Author details}

'Department of Basic Science of Stomatology, The Affiliated Stomatological Hospital of Nanjing Medical University, 136\# Hanzhong Road, Nanjing 210029, Jiangsu, China. ${ }^{2}$ Jiangsu Province Key Laboratory of Oral Diseases, Nanjing Medical University, Nanjing, Jiangsu, China. ${ }^{3}$ Jiangsu Province Engineering Research Center of Stomatological Translational Medicine, Nanjing, Jiangsu, China.

Received: 15 September 2021 Accepted: 19 January 2022

Published online: 05 February 2022

References

1. Siegel RL, Miller KD, Jemal A. Cancer statistics, 2020. CA Cancer J Clin. 2020;70(1):7-30

2. Bray F, Ferlay J, Soerjomataram I, Siegel RL, Torre LA, Jemal A. Global cancer statistics 2018: GLOBOCAN estimates of incidence and mortality worldwide for 36 cancers in 185 countries. CA Cancer J Clin. 2018;68(6):394-424.

3. Cramer JD, Burtness B, Le QT, Ferris RL. The changing therapeutic landscape of head and neck cancer. Nat Rev Clin Oncol. 2019;16(11):669-83.

4. von Witzleben A, Wang C, Laban S, Savelyeva N, Ottensmeier CH. HNSCC: tumour antigens and their targeting by immunotherapy. Cells. 2020;9(9):2103. 
5. Specenier P,Vermorken JB. Optimizing treatments for recurrent or metastatic head and neck squamous cell carcinoma. Expert Rev Anticancer Ther. 2018;18(9):901-15.

6. Chauhan SS, Kaur J, Kumar M, Matta A, Srivastava G, Alyass A, Assi J, Leong I, MacMillan C, Witterick I, et al. Prediction of recurrence-free survival using a protein expression-based risk classifier for head and neck cancer. Oncogenesis. 2015:4:e147.

7. Biomarkers Definitions Working G. Biomarkers and surrogate endpoints: preferred definitions and conceptual framework. Clin Pharmacol Ther. 2001;69(3):89-95.

8. Sears CR, Mazzone PJ. Biomarkers in Lung Cancer. Clin Chest Med. 2020;41(1):115-27.

9. Filella X, Foj L. Novel biomarkers for prostate cancer detection and prognosis. Adv Exp Med Biol. 2018;1095:15-39.

10. Burton C, Ma Y. Current trends in cancer biomarker discovery using urinary metabolomics: achievements and new challenges. Curr Med Chem. 2019;26(1):5-28.

11. Alsahafi E, Begg K, Amelio I, Raulf N, Lucarelli P, Sauter T, Tavassoli M. Clinical update on head and neck cancer: molecular biology and ongoing challenges. Cell Death Dis. 2019:10(8):540.

12. Shen Y, Liu J, Zhang L, Dong S, Zhang J, Liu Y, Zhou H, Dong W. Identification of potential biomarkers and survival analysis for head and neck squamous cell carcinoma using bioinformatics strategy: a study based on TCGA and GEO datasets. Biomed Res Int. 2019;2019:7376034.

13. Oliva M, Spreafico A, Taberna M, Alemany L, Coburn B, Mesia R, Siu LL. Immune biomarkers of response to immune-checkpoint inhibitors in head and neck squamous cell carcinoma. Ann Oncol. 2019;30(1):57-67.

14. Goossens N, Nakagawa S, Sun X, Hoshida Y. Cancer biomarker discovery and validation. TransI Cancer Res. 2015;4(3):256-69.

15. Frank D, Vince JE. Pyroptosis versus necroptosis: similarities, differences, and crosstalk. Cell Death Differ. 2019;26(1):99-114.

16. Xia X, Wang X, Cheng Z, Qin W, Lei L, Jiang J, Hu J. The role of pyroptosis in cancer: pro-cancer or pro-"host"? Cell Death Dis. 2019;10(9):650.

17. Jorgensen I, Miao EA. Pyroptotic cell death defends against intracellular pathogens. Immunol Rev. 2015;265(1):130-42.

18. Fang Y, Tian S, Pan Y, Li W, Wang Q, Tang Y, Yu T, Wu X, Shi Y, Ma P, et al. Pyroptosis: a new frontier in cancer. Biomed Pharmacother. 2020;121:109595.

19. Wang L, Qin X, Liang J, Ge P. Induction of pyroptosis: a promising strategy for cancer treatment. Front Oncol. 2021;11:635774.

20. Wang F, Liu W, Ning J, Wang J, Lang Y, Jin X, Zhu K, Wang X, Li X, Yang F, et al. Simvastatin suppresses proliferation and migration in non-small cell lung cancer via pyroptosis. Int J Biol Sci. 2018;14(4):406-17.

21. Yin XF, Zhang Q, Chen ZY, Wang HF, Li X, Wang HX, Li HX, Kang CM, Chu S, $\mathrm{Li}$ KF, et al. NLRP3 in human glioma is correlated with increased WHO grade, and regulates cellular proliferation, apoptosis and metastasis via epithelialmesenchymal transition and the PTEN/AKT signaling pathway. Int J Oncol. 2018;53(3):973-86.

22 Wang M, Jiang S, Zhang Y, Li P, Wang K. The multifaceted roles of pyroptotic cell death pathways in cancer. Cancers (Basel). 2019;11(9):1313.

23. Shao W, Yang Z, Fu Y, Zheng L, Liu F, Chai L, Jia J. The pyroptosis-related signature predicts prognosis and indicates immune microenvironment infiltration in gastric cancer. Front Cell Dev Biol. 2021;9:676485.

24. Ye Y, Dai Q, Qi H. A novel defined pyroptosis-related gene signature for predicting the prognosis of ovarian cancer. Cell Death Discov. 2021;7(1):71.

25. Cai J, Yi M, Tan Y, Li X, Li G, Zeng Z, Xiong W, Xiang B. Natural product triptolide induces GSDME-mediated pyroptosis in head and neck cancer through suppressing mitochondrial hexokinase-lotalota. J Exp Clin Cancer Res. 2021:40(1):190.

26. Shen $Y, L i X$, Wang $D$, Zhang L, Li X, Xia T, Shang $X$, Yang $X$, Su L, Fan $X$. Novel prognostic model established for patients with head and neck squamous cell carcinoma based on pyroptosis-related genes. Transl Oncol. 2021;14(12):101233

27. Karki R, Kanneganti TD. Diverging inflammasome signals in tumorigenesis and potential targeting. Nat Rev Cancer. 2019;19(4):197-214

28. Li H, Zhao XK, Cheng YJ, Zhang Q, Wu J, Lu S, Zhang W, Liu Y, Zhou MY, Wang $Y$, et al. Gasdermin D-mediated hepatocyte pyroptosis expands inflammatory responses that aggravate acute liver failure by upregulating monocyte chemotactic protein 1/CC chemokine receptor-2 to recruit macrophages. World J Gastroenterol. 2019;25(44):6527-40.
29. Tang R, Xu J, Zhang B, Liu J, Liang C, Hua J, Meng Q, Yu X, Shi S. Ferroptosis, necroptosis, and pyroptosis in anticancer immunity. J Hematol Oncol. 2020:13(1):110.

30. D'Arcy MS. Cell death: a review of the major forms of apoptosis, necrosis and autophagy. Cell Biol Int. 2019;43(6):582-92.

31. Jia C, Chen H, Zhang J, Zhou K, Zhuge Y, Niu C, Qiu J, Rong X, Shi Z, Xiao J, et al. Role of pyroptosis in cardiovascular diseases. Int Immunopharmacol. 2019;67:311-8.

32. Al Mamun A, Wu Y, Jia C, Munir F, Sathy KJ, Sarker T, Monalisa I, Zhou K, Xiao J. Role of pyroptosis in liver diseases. Int Immunopharmacol. 2020;84:106489.

33. Chen X, He WT, Hu L, Li J, Fang Y, Wang X, Xu X, Wang Z, Huang K, Han J. Pyroptosis is driven by non-selective gasdermin-D pore and its morphology is different from MLKL channel-mediated necroptosis. Cell Res. 2016:26(9):1007-20.

34. Nagarajan K, Soundarapandian K, Thorne RF, Li D, Li D. Activation of pyroptotic cell death pathways in cancer: an alternative therapeutic approach. Transl Oncol. 2019;12(7):925-31.

35. Zhou S, Gravekamp C, Bermudes D, Liu K. Tumour-targeting bacteria engineered to fight cancer. Nat Rev Cancer. 2018;18(12):727-43.

36. Feng $X$, Luo $Q$, Zhang H, Wang $H$, Chen W, Meng G, Chen F. The role of NLRP3 inflammasome in 5-fluorouracil resistance of oral squamous cell carcinoma. J Exp Clin Cancer Res. 2017;36(1):81.

37. Cheng AL, Kang YK, Chen Z, Tsao CJ, Qin S, Kim JS, Luo R, Feng J, Ye S, Yang TS, et al. Efficacy and safety of sorafenib in patients in the Asia-Pacific region with advanced hepatocellular carcinoma: a phase III randomised, double-blind, placebo-controlled trial. Lancet Oncol. 2009;10(1):25-34.

38. Zhou JG, Liang B, Jin SH, Liao HL, Du GB, Cheng L, Ma H, Gaipl US. Development and validation of an RNA-Seq-based prognostic signature in neuroblastoma. Front Oncol. 2019:9:1361.

39. Kim SK, Kim HJ, Park JL, Heo H, Kim SY, Lee SI, Song KS, Kim WH, Kim YS. Identification of a molecular signature of prognostic subtypes in diffuse-type gastric cancer. Gastric Cancer. 2020;23(3):473-82.

40. Yao Y, Yan Z, Lian S, Wei L, Zhou C, Feng D, Zhang Y, Yang J, Li M, Chen Y. Prognostic value of novel immune-related genomic biomarkers identified in head and neck squamous cell carcinoma. I Immunother Cancer. 2020;8(2):e000444.

41. Wang J, Chen X, Tian Y, Zhu G, Qin Y, Chen X, Pi L, Wei M, Liu G, Li Z, et al. Six-gene signature for predicting survival in patients with head and neck squamous cell carcinoma. Aging. 2020;12(1):767-83.

42. Man SM, Karki R, Kanneganti TD. Molecular mechanisms and functions of pyroptosis, inflammatory caspases and inflammasomes in infectious diseases. Immunol Rev. 2017:277(1):61-75.

43. VandeWalle L, Lamkanfi M. Pyroptosis. Curr Biol. 2016;26(13):R568-72.

44. Gnjatic S, Bronte V, Brunet LR, Butler MO, Disis ML, Galon J, Hakansson LG, Hanks BA, Karanikas V, Khleif SN, et al. Identifying baseline immune-related biomarkers to predict clinical outcome of immunotherapy. I Immunother Cancer. 2017:5:44

45. Etxebeste-Mitxeltorena M, Del Rincon-Loza I, Martin-Antonio B. Tumor secretome to adoptive cellular immunotherapy: reduce me before I make you my partner. Front Immunol. 2021;12:717850.

46 Singla DK, Johnson TA, TavakoliDargani Z. Exosome treatment enhances anti-inflammatory M2 macrophages and reduces inflammation-induced pyroptosis in doxorubicin-induced cardiomyopathy. Cells. 2019:8(10):1224.

47. Swanson KV, Deng M, Ting JP. The NLRP3 inflammasome: molecular activation and regulation to therapeutics. Nat Rev Immunol. 2019;19(8):477-89.

48. Lu F, Zhao Y, Pang Y, Ji M, Sun Y, Wang H, Zou J, Wang Y, Li G, Sun T, et al. NLRP3 inflammasome upregulates PD-L1 expression and contributes to immune suppression in lymphoma. Cancer Lett. 2021;497:178-89.

49. Ren N, Jiang T, Wang C, Xie S, Xing Y, Piao D, Zhang T, Zhu Y. LncRNA ADAMTS9-AS2 inhibits gastric cancer (GC) development and sensitizes chemoresistant GC cells to cisplatin by regulating miR-223-3p/NLRP3 axis. Aging (Albany NY). 2020;12(11):11025-41.

50. Wei Q, Mu K, Li T, Zhang Y, Yang Z, Jia X, Zhao W, Huai W, Guo P, Han L. Deregulation of the NLRP3 inflammasome in hepatic parenchymal cells during liver cancer progression. Lab Invest. 2014;94(1):52-62.

\section{Publisher's Note}

Springer Nature remains neutral with regard to jurisdictional claims in published maps and institutional affiliations. 